\title{
A rare case of a completely thrombosed bilobed giant intracranial aneurysm of the anterior cerebral artery with spontaneous parent vessel thrombosis: case report
}

\author{
Mehdi Chihi ${ }^{*}$ (D, Ramazan Jabbarli', Oliver Gembruch', Sarah Teuber-Hanselmann², Marvin Darkwah Oppong1, \\ Daniela Pierscianek ${ }^{1}$, Alexander Radbruch ${ }^{3}$, Martin Glas ${ }^{4}$, Mark Stettner ${ }^{4}$ and Ulrich Sure ${ }^{1}$
}

\begin{abstract}
Background: A huge spherical intracranial mass can sometimes be misdiagnosed, due to the lack of typical radiographic features. Thrombosed giant intracranial aneurysms (GIAs) are an uncommon but still a possible differential diagnosis that must be kept in mind to guarantee the best surgical approach and resection of the lesion. We describe an extremely rare case of a huge bifrontal mass mimicking a cystic echinococcosis, in which the surgery unveiled a completely thrombosed GIA of the left anterior cerebral artery (ACA).

Case presentation: A 61-year-old patient complained about intermittent weakness of the right leg, mild holocephalic headache, beginning cognitive deficits and lethargy. Magnetic resonance imaging (MRI) showed a huge partially calcified and bilobed frontal mass with peripheral edema. Based on a time-resolved angiography with interleaved Stochastic trajectories MRI (TWIST-MRI), a vascular origin of the lesion was considered unlikely. Therefore, the surgery was performed under the suspicion of a cystic echinococcosis but revealed a bilobed GIA of the left ACA with a parent vessel thrombosis. Although only a limited left frontal craniotomy was performed, a proximal control of the parent vessel could be ensured, and the aneurysm was successfully clipped. The patient showed postoperatively no new neurological deficits.

Conclusions: Completely thrombosed GIAs with parent vessel thrombosis are rare lesions that might be misdiagnosed if typical radiographic features are missing. Thus, in case of an intracranial spherical mass with signs of intralesional hemorrhage and mural calcifications, presence of a completely thrombosed GIA should be considered as a possible differential diagnosis.
\end{abstract}

Keywords: Giant intracranial aneurysm, Intraluminal thrombosis, Clipping, Parent vessel

\section{Background}

Giant intracranial aneurysms (GIAs) are rare and heterogeneous lesions with complex vascular anatomy [1] and represent almost $5 \%$ of intracranial aneurysms (IAs) [2]. They typically become symptomatic between the 4th and the 7th decade, with a female to male ratio ranging from 1:1 to 3:1 [2]. The incidence of intraluminal thrombosis ranges from 10 to $30 \%$ of the cases, [3] however, a complete aneurysmal thrombosis is extremely rare [4]. The majority of these aneurysms are located in the internal carotid artery (ICA) and in the middle cerebral artery (MCA), the anterior cerebral artery (ACA) being an exceptional location [1].

In this report, we present an unusual case of a completely thrombosed GIA of the left ACA, that was preoperatively misdiagnosed because of the lack of typical radiographic features.

\footnotetext{
*Correspondence: mehdi.chihi@uk-essen.de

'Department of Neurosurgery, University Hospital Essen, University of

Duisburg-Essen, Essen, Germany

Full list of author information is available at the end of the article
}

(c) The Author(s). 2019 Open Access This article is distributed under the terms of the Creative Commons Attribution 4.0 International License (http://creativecommons.org/licenses/by/4.0/), which permits unrestricted use, distribution, and 


\section{Case presentation}

A 61-year-old male patient was referred to our neurosurgical department with a 10-day history of an intermittent weakness of the right leg, mild holocephalic headache, beginning cognitive deficits and lethargy. A recent stay in America, China and East Africa was reported. There was no fever and no weight loss. Physical examination showed an ataxic gait. During the hospital stay, the patient developed a focal seizure of the right leg, which was treated with levetiracetam.

\section{Radiological findings(Fig. 1)}

Magnetic resonance imaging (MRI) obtained from the referring hospital reported two well-defined frontal bihemispheric masses, 54 and $40 \mathrm{~mm}$ in diameter, with a slight perifocal edema. The patient was admitted to the intermediate care unit and a computed tomography angiography was performed. The latter showed two large lesions in both frontal regions, partly inhomogeneous, with central hyperdense parts and with partial mural calcifications. A thorax and abdomen CT showed no tumor manifestation. A time-of-flight MRI (TOF-MRI) demonstrated the right ACA shifted to the lateral wall of the lesion, whereas only the proximal left ACA (A1-segment) was displayed. Time-resolved angiography with interleaved stochastic trajectories MRI (TWIST-MRI) was also performed and showed neither contrast enhancement nor intraluminal filling of both masses.

\section{Laboratory findings}

Blood and cerebrospinal fluid (CSF) screening presented no signs of infection. There were no tumor cells in the CSF. Parasitological examination of blood and CSF was performed. A low antibody concentration against Echinococcus multilocularis was detected in the patient's CSF (1:20).

\section{Surgical treatment (Fig. 2)}

Because of the recent foreign stay in East Africa and the above-mentioned CSF results, the diagnosis of a cerebral cystic echinococcosis was suspected. Consequently, no digital subtraction angiography (DSA) was performed, as a GIA in this case was besides radiologically considered extremely unlikely, and a microsurgical resection of the lesion was indicated. Thus, a left frontal craniotomy was performed. The preparation showed a very hard cystic lesion with vasa vasorum at the lesion wall evoking the

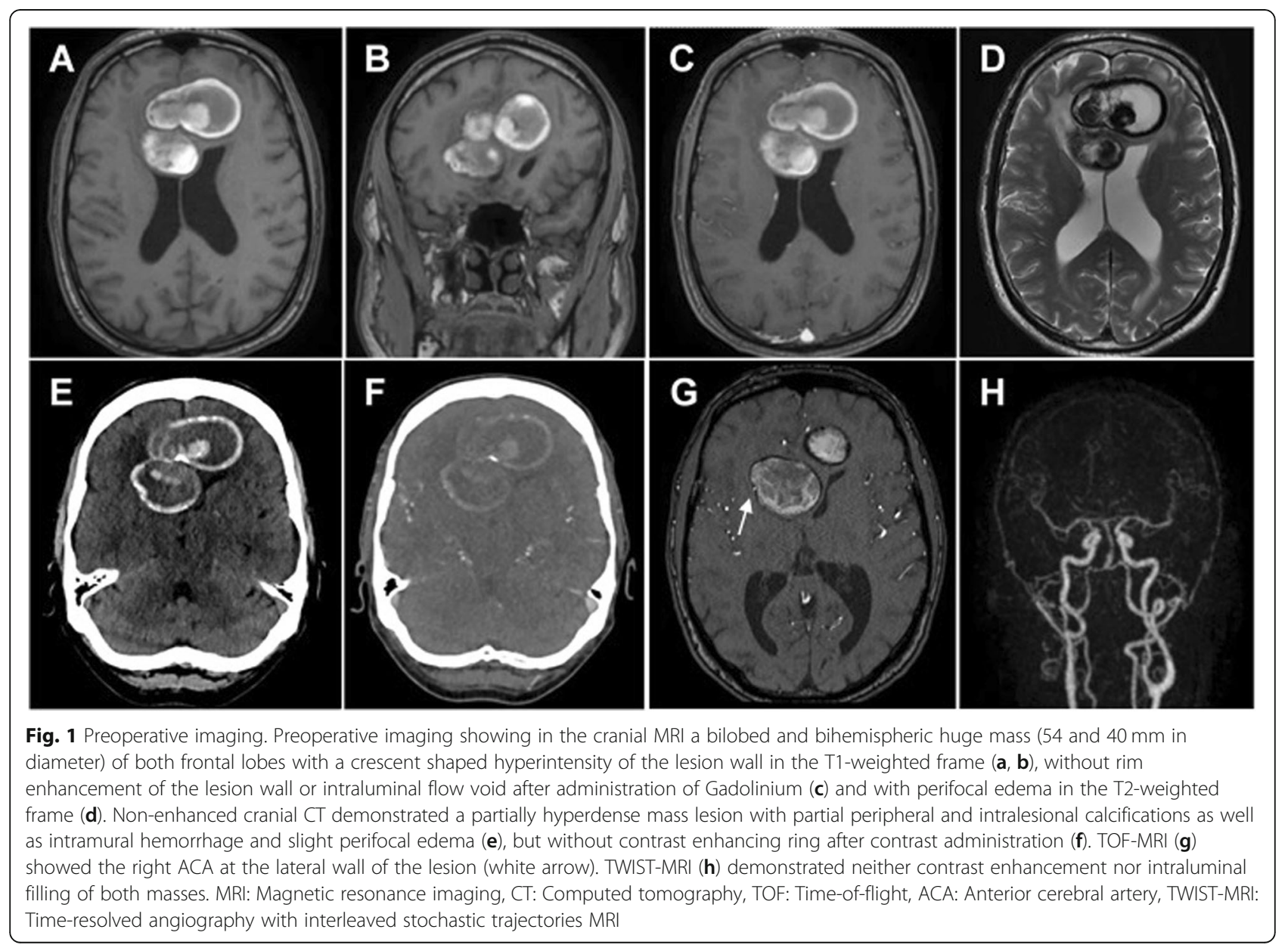




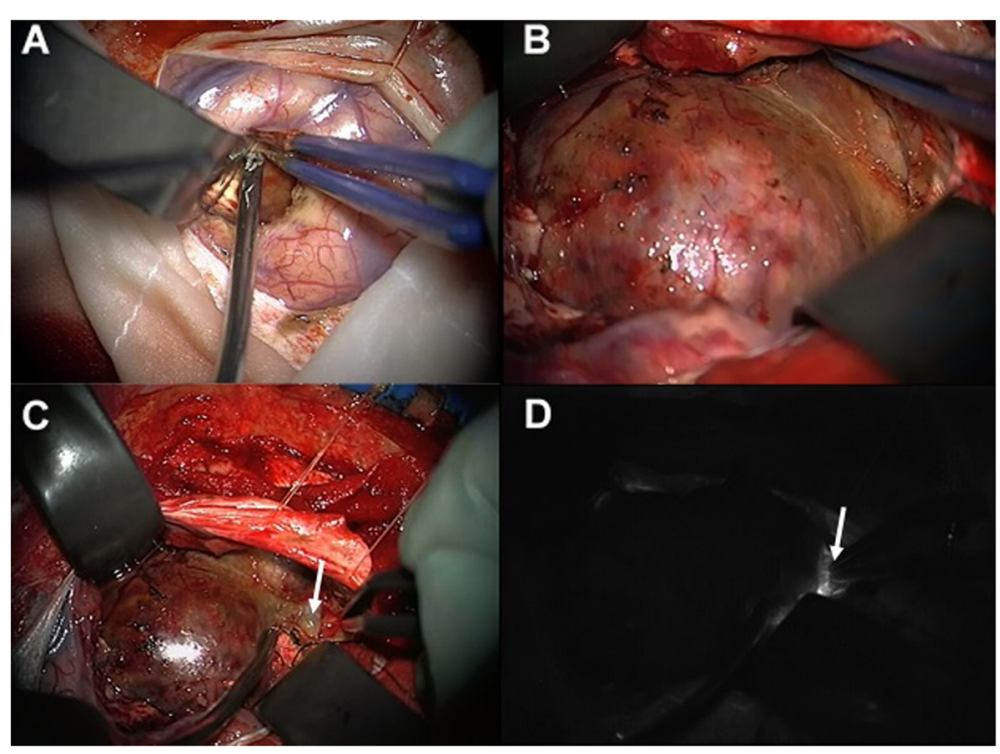

Fig. 2 Intraoperative photographs. After left frontal craniotomy and corticotomy, a hard-cystic lesion was found (a) with vasa vasorum at its wall (b). Meticulous dissection in all direction was performed until the parent vessel was found (c, white arrow). The Indocyanine green angiography showed only a very low blood flow in the parent artery (d, white arrow)

differential diagnosis of a thrombosed aneurysm. The preparation of the GIA was most difficult due to the absence of a proximal control through the performed frontal craniotomy. After a careful and meticulous dissection, the lesion could be finally prepared in all directions. Furthermore, the aneurysmal parent vessel was found. The intraoperative Indocyanine green angiography demonstrated a very low blood flow in this thrombosed vessel. After clipping of the parent vessel, the two aneurysmal sacs were opened, and the thrombi and calcifications were resected.

\section{Histopathology (Fig. 3)}

Histopathological examination revealed wall fragments of an artery of large caliber adjacent to thrombotic material. The vessel wall was irregularly thickened with eccentric fibrosis or abnormal wall thinning; an internal elastic lamina was seen only focally. Hemosiderin deposits, calcifications and inflammatory infiltrates were detectable within the remnants of the vessel wall.

\section{Postoperative course and follow-up (Fig. 4)}

Postoperatively, the patient did well and had no neurological deficits. A DSA showed no aneurysmal remnant after clipping. A collateral circulation coming from both MCAs and the posterior circulation was detected. At the 6-month follow-up, the patient recovered from the operation and could walk without assistance.

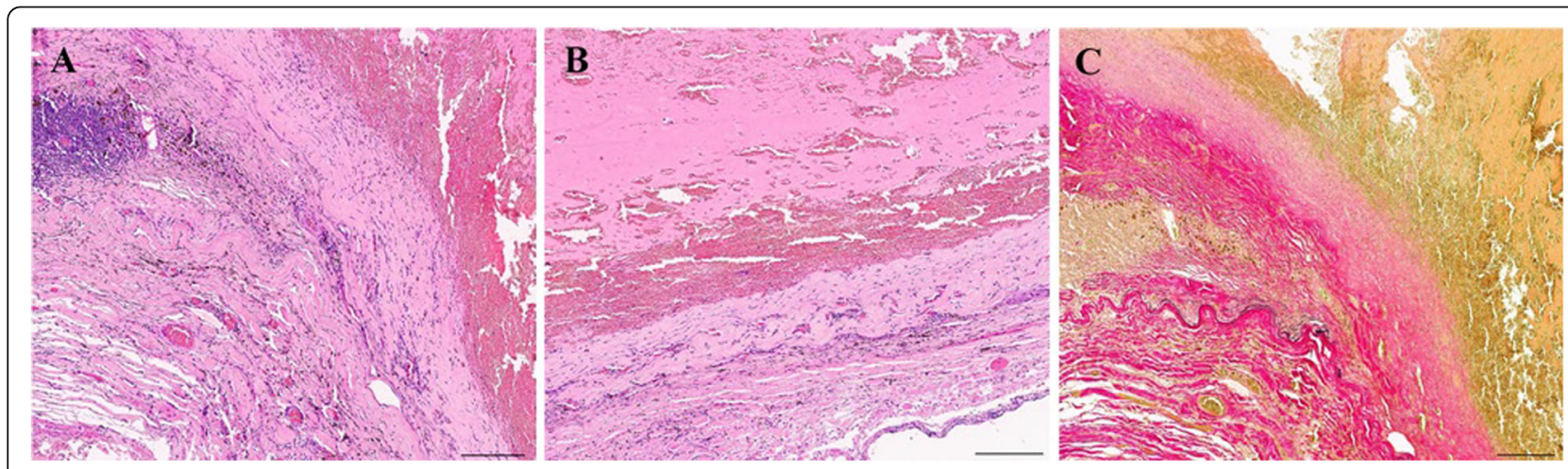

Fig. 3 Histopathological findings. Hematoxylin-eosin staining revealed wall fragments of a large caliber artery with eccentric fibrosis, inflammatory infiltrates and hemosiderin deposits (a) or abnormal wall thinning (b) adjacent to thrombotic material. The internal elastic lamina ends abruptly (Elastica-van-Gieson staining; c Scale bar: $200 \mu \mathrm{m}$ ) 


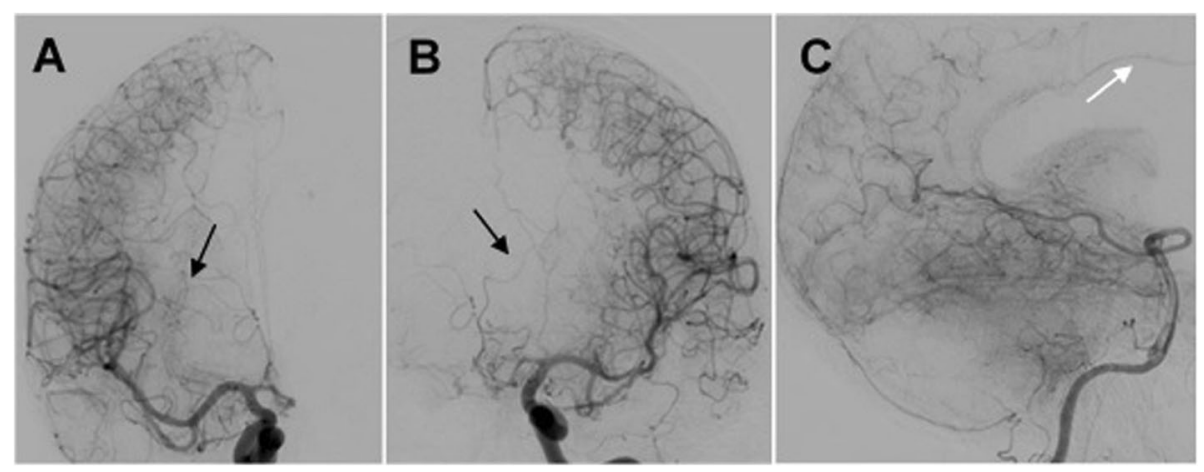

Fig. 4 Postoperative imaging. Postoperative DSA showing no aneurysmal remnant after clipping the bilobed GIA of the anterior circulation. A collateral circulation coming from both MCAs (a and $\mathbf{b}$, black arrows) and the posterior circulation (white arrow) was detected (c). DSA: Digital substraction angiography, GIA: Giant intracranial aneurysm, MCA: Middle cerebral artery

\section{Discussion}

The natural history of GIAs is featured with three pathological events: spontaneous thrombosis, growth leading to a mass effect and rupture causing a subarachnoid hemorrhage [2]. These are characterized by a high mortality rate, essentially for aneurysms located on the posterior circulation, [5] as the rupture rate in 5 years ranges between 40 and 50\% [6]. Most of the time, as in our case, GIAs are diagnosed in the course of a pseudotumoral syndrome, where the mass effect of the IA leads to a gradual neurologic deterioration [7].

Complete intraluminal thrombosis is uncommon and occurs especially in large and giant cerebral aneurysms in $13-20 \%$ of cases [4]. This phenomenon is explained by endothelial damage due to hemodynamic stress on the aneurysmal wall [4]. CT characteristics of thrombosed IAs are peripheral ring enhancement, curvilinear mural calcification, intraluminal mixed high-density calcification and the presence of "target sign" [8]. On the other hand, MRI features include "onion skin" appearance, luminal flow void, luminal enhancement and perianeurysmal edema on T2weighted sequences [9]. In our case, neither intraluminal flow void nor peripheral contrast enhancement was detected on MRI and CT. Although all radiological examinations -excluding DSA- were carried out, a misdiagnosis was made because of the lack of typical radiographic features.

Moreover, the left A2-segment was not detected on the TOF-MRI. Based on this MRI feature and on the intraoperative fact that the parent vessel was probably coming from the left ACA, spontaneous occlusion of the parent artery (left A2-segment) harboring the GIA was suspected. Indeed, although this scenario is extremely rare, [4] it would probably explain the absence of intraluminal flow void and absence of enhancement of the aneurysmal parent vessel on both TOF- and TWISTMRI. In fact, the mechanism of the spontaneous thrombosis of an aneurysm and its parent artery is not known [4]. Theories of local stretching, compression and distortion of the ICA could explain the occurrence of this complication in IAs of the cavernous segment, due to the presence of dural folds and bony structures at this location [10]. In our case, compression of the parent vessel by a growing partially thrombosed aneurysm, or retrograde propagation of the thrombus, [11] leading to an acute parent artery thrombosis and subsequently to a complete thrombosis of the aneurysm, are two possible mechanisms.

In this way, because of the limits of imaging, in front of a huge intracranial mass presenting with mural calcification and signs of hemorrhage, a completely thrombosed GIA must be considered as a differential diagnosis and must not be ruled out until the operation is performed. Preoperatively, a DSA is always helpful and must be included in the preoperative workup to visualize the parent vessel of the IA and plan the operation. Even though, in the case of a completely thrombosed aneurysm, DSA could be negative [12]. Furthermore, a well-trained vascular neurosurgeon must be available on the day of operation, especially in non-high volume vascular centers. In our case, although the diagnosis of GIA was made intraoperatively, careful and meticulous preparation of the aneurysm allowed us to find the parent artery and have a proximal control before clipping the aneurysm.

\section{Conclusions}

Completely thrombosed GIAs with parent vessel thrombosis are rare lesions that might be misdiagnosed if typical radiographic features are missing. Thus, in case of an intracranial spherical mass with signs of intralesional hemorrhage and mural calcifications, presence of a completely thrombosed GIA should be considered as a possible differential diagnosis.

\section{Abbreviations}

ACA: Anterior cerebral artery; CSF: Cerebrospinal fluid; CT: Computer tomography; DSA: Digital subtraction angiography; GIA: Giant intracranial 
aneurysm; IA: Intracranial aneurysm; ICA: Internal carotid artery; MCA: Middle cerebral artery; MRI: Magnetic resonance imaging; TOF-MRI: Time-of-flight MRI; TWIST-MRI: Time-resolved angiography with interleaved stochastic trajectories MRI

\section{Acknowledgments}

We acknowledge support by the Open Access Publication Fund of the University of Duisburg-Essen

\section{Authors' contributions}

MC Conceptualized and designed the study; acquired, analyzed and interpreted the data; drafted the article; was the major contributor in writing the manuscript; and approved the final version to be submitted. RJ Analyzed and interpreted the data; revised it critically for important intellectual content; and approved the final version to be submitted. OG Analyzed and interpreted the data; reviewed and revised the final version to be submitted. STH Performed the histological examination of the aneurysm; provided the histological figures; reviewed and revised the final version to be submitted. MDO Analyzed and interpreted the data; reviewed and revised the final version to be submitted. DP Analyzed and interpreted the data; revised it critically for important intellectual content; and approved the final version to be submitted. AR Interpreted and evaluated the imaging study; revised the manuscript for important intellectual content; and approved the final version to be submitted. MS Performed the diagnostic work up in the department of neurology; and approved the final version to be submitted. MG Performed and supervised the diagnostic work up in the department of neurology; revised the manuscript for important intellectual content; and approved the final version to be submitted. US Conceptualized and designed the study, operated the patient, revised the manuscript critically for important intellectual content; and approved the final version to be submitted. Al authors read and approved the final manuscript.

\section{Funding}

This case report did not receive any specific grant from funding agencies in the public, commercial, or not-for-profit sectors.

\section{Availability of data and materials}

All data generated or analyzed during this study are included in this published article.

\section{Ethics approval and consent to participate}

The case report has been carried out in accordance with The Code of Ethics of the World Medical Association (Declaration of Helsinki).

\section{Consent for publication}

A written informed consent was obtained from the patient.

\section{Competing interests}

The work reported in the present manuscript was not supported financially and no conflicts of interest for any of the authors needs to be mentioned.

\section{Author details}

${ }^{1}$ Department of Neurosurgery, University Hospital Essen, University of Duisburg-Essen, Essen, Germany. ${ }^{2}$ Institute of Neuropathology, University Hospital Essen, University of Duisburg-Essen, Essen, Germany. ${ }^{3}$ Institute of Diagnostic and Interventional Radiology and Neuroradiology, University Hospital Essen, University of Duisburg-Essen, Essen, Germany. ${ }^{4}$ Department of Neurology, University Hospital Essen, University of Duisburg-Essen, Essen, Germany.

Received: 8 August 2019 Accepted: 13 November 2019 Published online: 23 November 2019

\section{References}

1. Nurminen V, Lehecka M, Chakrabarty A, Kivisaari R, Lehto H, Niemelä M, et al. Anatomy and morphology of giant aneurysms-angiographic study of 125 consecutive cases. Acta Neurochir. 2014;156(1):1-10.

2. Lonjon M, Pennes F, Sedat J, Bataille B. Epidemiology, genetic, natural history and clinical presentation of giant cerebral aneurysms. Neurochirurgie. 2015;61 (6):361-5.
3. dos Santos MLT, Spotti AR, dos Santos RMT, Borges MA, Ferrari AF, Colli BO, et al. Giant intracranial aneurysms: morphology and clinical presentation. Neurosurg Rev. 2013;36(1):117-22.

4. Das KK, Singh G, Pandey S, Bhaisora KS, Jaiswal A, Behari S. Completely Thrombosed Giant intracranial aneurysm with spontaneous thrombosis of the parent artery: is it Nature's divine intervention and a self-cure? World Neurosurg. 2018;118:132-8.

5. Drake CG. Giant intracranial aneurysms: experience with surgical treatment in 174 patients. Clin Neurosurg. 1979;26:12-95.

6. Wiebers DO, Investigators ISoUIA. Unruptured intracranial aneurysms: natural history, clinical outcome, and risks of surgical and endovascular treatment. Lancet. 2003;362(9378):103-10.

7. Choi IS, David C. Giant intracranial aneurysms: development, clinical presentation and treatment. Eur J Radiol. 2003;46(3):178-94.

8. Schubiger O, Valavanis A, Wichmann W. Growth-mechanism of giant intracranial aneurysms; demonstration by CT and MR imaging. Neuroradiology. 1987;29(3):266-71.

9. Teng MM-H, Qadri SN, Luo C-B, Lirng J-F, Chen S-S, Chang C-Y. MR imaging of giant intracranial aneurysm. J Clin Neurosci. 2003;10(4):460-4.

10. Perrini P, Bortolotti C, Wang H, Fraser K, Lanzino G. Thrombosed giant intracavernous aneurysm with subsequent spontaneous ipsilateral carotid artery occlusion. Acta Neurochir. 2005;147(2):215-7.

11. Whittle IR, Williams DB, Halmagyi GM, Besser M. Spontaneous thrombosis of a giant intracranial aneurysm and ipsilateral internal carotid artery: case report. J Neurosurg. 1982;56(2):287-9.

12. Trungu S, Bruzzaniti P, Forcato S, Cimatti M, Raco A. Completely thrombosed distal middle cerebral artery aneurysm mimicking a cavernous angioma: case report and review of the literature. World Neurosurg. 2017; 103:955.e1-4.

\section{Publisher's Note}

Springer Nature remains neutral with regard to jurisdictional claims in published maps and institutional affiliations.
Ready to submit your research? Choose BMC and benefit from:

- fast, convenient online submission

- thorough peer review by experienced researchers in your field

- rapid publication on acceptance

- support for research data, including large and complex data types

- gold Open Access which fosters wider collaboration and increased citations

- maximum visibility for your research: over $100 \mathrm{M}$ website views per year

At $\mathrm{BMC}$, research is always in progress.

Learn more biomedcentral.com/submissions 